\title{
On the use of quasi-equidistant source points over the sphere surface for the method of fundamental solutions
}

\author{
António Araújo ${ }^{\mathrm{a}, \mathrm{b}, \mathrm{c}}$, Pedro Serranho, ${ }^{\mathrm{a}, \mathrm{d}, \mathrm{e}, *}$ \\ ${ }^{a}$ Mathematics Section, Department of Science and Technology, Universidade Aberta, Portugal \\ ${ }^{b}$ Centro de Matemática, Aplicacoes Fundamentais e Investigação Operacional \\ ${ }^{c}$ Centro de Investigação em Artes e Comunicacao. \\ ${ }^{d}$ Coimbra Institute for Biomedical Imaging and Translational Research, Faculty of Medicine, University of Coimbra, Coimbra, Portugal \\ ${ }^{e}$ Center for Computational and Stochastic Mathematics, University of Lisbon, Lisbon, Portugal
}

\begin{abstract}
The method of fundamental solutions is broadly used in science and engineering to numerically solve the direct time-harmonic scattering problem. In 2D the choice of source points is usually made by considering an inner pseudo-boundary over which equidistant source points are placed. In 3D, however, this problem is much more challenging, since, in general, $n$ equidistant points over a closed surface do not exist. In this paper we discuss a method to obtain a quasi-equidistant point distribution over the unit sphere surface, giving rise to a Delaunay triangulation that might also be used for other boundary element methods. We give theoretical estimates for the expected distance between points and the expect area of each triangle. We illustrate the feasibility of the proposed method in terms of the comparison with the expected values for distance and area. We also provide numerical evidence that this points distribution leads to a good conditioning of the linear system associated with the direct scattering problem, being therefore an adequated choice of source points for the method of fundamental solutions.
\end{abstract}

Keywords: Method of fundamental solutions, source points, equidistant points, triangulation 2010 MSC: $65 \mathrm{~N} 80$

\section{Introduction}

The method of fundamental solutions (MFS) is a broadly used method for solving elliptic partial differential equations. Its easy formulation and computational implementation combined with good numerical results makes it very popular amongst the mathematical and engineering community $[1,2,3,4]$.

Given an homogeneous partial differential equation to be satisfied in an open set $D$ and the corresponding fundamental solution $\Phi$ of the partial differential equation, the MFS consists in approximating the solution $u$ as a sum of fundamental solutions as

$$
u(x) \approx \sum_{j=1}^{n_{s}} \omega_{j} \Phi\left(x, s_{j}\right)
$$

using a set of proper source points $s_{j}, j=1,2, \ldots, n_{s}$, where the weights $\omega_{j}$ are usually defined by solving a linear system according to other information on the solution $u$ such as a boundary condition, for instance. Density results of fundamental solutions over the boundary can be seen, for instance, in [5]. We will discuss in detail how the method 
can be applied to the exterior Helmholtz equation later in section 4. For now, we focus on the fact that by the definition of fundamental solution the ansatz (1) automatically satisfies the homogeneous partial differential equation in some smooth domain $D$ if the source points satisfy $s_{j} \notin \bar{D}, j=1,2, \ldots, n_{s}$. However, the choice of the source points has two main implications for the MFS, since it usually influences the conditioning of the linear system to determine the weights $\omega_{j}$ and the accuracy of the approximation of the form (1). Ideally one should aim to a compromise between both, since in [6] it was shown that the choice of the source points may lead the numerical approximation to vary from excellent to numerically unsolvable, in terms of the quality of the approximation to the solution of a problem. Usually, the numerical approximation for the exterior problem (resp., interior problem) by MFS considers the source points lying on an inner (resp., outer) pseudo-boundary obtained by moving the smooth boundary of the domain $D$ in the normal direction (see [7], for a more general setting in 2D). The pseudo-boundary should not be to close to the real boundary due to the ill-conditioning, nor too far, in order to get good accuracy. In 2D, it is easy to find equidistant points in the pseudo-boundary and numerical experiments have shown that usually these lead to good results. Also, several papers have been written concerning stability, convergence, efficiency and conditioning of the MFS with source points in the 2D unit disk [7, 8, 9].

However, in $3 \mathrm{D}$ the problem of finding equidistant points on a surface has in general no solution (as we will discuss later in section 2) and the problem of finding quasi-equidistant points in also not trivial. Therefore, the source points are usually chosen in other ways, as for instance, equidistant in the parametrization space, which in the case of a sphere surface leads to a equiangular distribution of points but not to equidistant points. In [10] a more complex choice of source points over the sphere surface was chosen, namely the icosahedral tesselation of the sphere surface that we will also consider in this work for comparison purposes. In [11] a method is used to improve the conditioning of the MFS, though through a preconditioning technique rather than the optimization of the location of points. The problem at hand was also discussed in terms os accuracy of solution in 2D and 3D in [12] for the Laplace and biharmonic equations. Also, in [13] an application of MFS is made using the unit sphere. Some examples of the application of the MFS in 3D are $[1,4,14,15,16,17,18,19,20]$.

In this paper we propose a method to determine quasi-equidistant points over the unit sphere surface (from this point on designated simply by sphere), showing that the use of these as source points for the MFS leads to precise solutions and good conditioning. These can then be adapted to other parametrizable surfaces in 3D, as illustrated in section 5.2.2. We will illustrate that the proposed method generates quasi-equidistant points and that the respective Delaunay triangulation is composed by triangles with similar areas. We will also estimate the expected distance between points and the expected area of the triangle, in a quasi-equidistant point distribution.

The paper is organized as follows. In section 2 we discuss estimates on the mean distance between quasiequidistant points over the sphere and the expected area of each triangle of the respective triangulation. In section 3 we propose a method to obtain $n$ quasi-equidistant points over the sphere, for any given $n$. In section 4 we address the application of the MFS to the Helmholtz equation in a exterior domain. In section 5 we present the numerical results for the obtained quasi-equidistant points and illustrate its use for MFS source points, showing the good conditioning of the numerical method applied to the exterior Helmholtz problem. Finally, in section 6 we summarize the results and discuss future perspectives on this work.

\section{Estimates for quasi-equidistant points in the sphere}

As a reminder, we stress that for simplicity we designate by sphere the unit sphere surface $S^{2}$.

We first need to define what we mean by uniformly distributed equidistant points over the sphere.

Definition 1 (Uniformly distributed equidistant points over the sphere). We define a set of $n$ points $\hat{x}_{1}, \hat{x}_{2}, \ldots, \hat{x}_{n}$ over the unit sphere $S^{2}$, that is,

$$
\left\{\hat{x}_{i}:\left\|\hat{x}_{i}\right\|_{2}=1, \forall i=1,2, \ldots, n\right\},
$$

to be uniformly distributed equidistant points if and only if these are the vertex of a geometric solid with identical regular geometric faces.

We start by noting that for the five Platonic solids it is possible to have uniformly distributed points over the sphere, which makes the problem of having $n$ uniformly distributed equidistant points in the sphere solvable for $n=$ $4,6,8,12,20$. However, for other values of $n$, specially in the case of interest of $n$ large, the problem has no solution. 
In this way, for other values of $n$, one should aim only at uniformly distributed quasi-equidistant points, since equidistant points are not available.

Definition 2 (Uniformly distributed quasi-equidistant points over the sphere). We define a set of $n$ points $\hat{x}_{1}, \hat{x}_{2}, \ldots, \hat{x}_{n}$ over the sphere $S^{2}$, that is,

$$
\left\{\hat{x}_{i}:\left\|\hat{x}_{i}\right\|_{2}=1, \forall i=1,2, \ldots, n\right\},
$$

to be uniformly distributed $\varepsilon$-quasi-equidistant points if and only if there exists a distance $d$ and an $\varepsilon>0$

1. such that

$$
\forall i, k \in\{1, \ldots, n\},\left\|\hat{x}_{k}-\hat{x}_{i}\right\| \geq d
$$

2. for any natural $m$ and any point $x \in S^{2}$ there exists a natural

$$
q \in\{1,2, \ldots, m-1\} \cup\{m+1, \ldots, n\}
$$

such that

$$
\left\|x-\hat{x}_{q}\right\| \leq d+\varepsilon .
$$

Remark 3. Conditions (2)-(3) are easily satisfied for any set of points $\hat{x}_{1}, \hat{x}_{2}, \ldots, \hat{x}_{n}$ for small enough $d$ and large enough $\varepsilon$. However, to our goal, we are interested in $\varepsilon$ as small as possible and therefore, $d$ as large as possible.

After these definitions, we are interested in theoretical estimates on the distance $d$ with respect to the number of points $n$.

We note the following:

Theorem 4. All triangulations of the sphere with $n$ points have exactly $2 n-4$ triangles and $3 n-6$ sides.

proof: The Euler characteristic of the sphere is $\chi=2$, so by Euler's theorem, $2=v-e+f$, where $v=n, e, f$ are the numbers of vertices, edges, and faces of the triangulation, respectively. Furthermore, since each triangle has 3 sides but each side is shared by 2 triangles exactly, we have $3 f=2 e$. Hence $f=2 v-4$, and $e=(3 / 2)(2 v-4)=3 v-6$.

From theorem 4 we know that, if a regular $n$-point triangulation of the sphere could be obtained, then each triangle would have area equal to

$$
A_{n}=4 \pi /(2 n-4) .
$$

Of course, this is impossible in general. Except in 4 of the 5 cases that correspond to the platonic solids, a general triangulation will have triangles with different areas. But in the other cases the spread of the distribution of the areas around $A_{n}$ serves as a measure for the degree of irregularity of the triangulation. We will measure this spread around $A_{n}$ by numerical calculation of the standard deviation

$$
\sigma_{A}(\Delta)=\sqrt{\frac{1}{2 n-4} \sum_{i=1}^{2 n-4}\left(A_{\Delta}(i)-A_{n}\right)^{2}},
$$

where $A_{\Delta}(i)$ is the area of the $i$-th triangle of the $n$-point triangulation $\Delta$.

Let $d_{n}$ be the length of the sides of the equilateral triangle of area $A_{n}$. This would be the side length of a regular triangulation. Again, since this triangulation is in general impossible, the spread of the side lengths around $d_{n}$ serves as a measure of deviation from the ideal case. We can also measure it through the standard error

$$
\sigma_{d}(\Delta)=\sqrt{\frac{1}{3 n-6} \sum_{i=1}^{3 n-6}\left(d_{\Delta}(i)-d_{n}\right)^{2}},
$$

where $d_{\Delta}(i)$ is the length of side $i$ of the $n$-point triangulation $\Delta$. Let us then calculate the value of $d_{n}$. 
Theorem 5. If $\Delta$ is a regular triangulation of the sphere by equilateral triangles then the length of the sides of the triangles is equal to

$$
d_{n}=\arccos \left[\frac{\cos \left(\alpha_{n}\right)+\cos ^{2}\left(\alpha_{n}\right)}{\sin ^{2}\left(\alpha_{n}\right)}\right], \text { where } \alpha_{n}=\frac{\pi}{3}\left(\frac{1}{1-2 / n} .\right)
$$

proof: We know from spherical trigonometry that for a spherical triangle with angles $A, B, C$ and sides $a, b, c$ (as in figure 1), the area of the triangle is equal to $A_{\Delta}=A+B+C-\pi$, and the sides of the triangle relate to the angles by the expression $\cos (a)=(\cos (A)+\cos (B) \cos (C)) /(\sin (B) \sin (C))$. For equilateral triangles we have $A=B=C=\alpha_{n}$ for some $\alpha_{n}$, hence $A_{\Delta}=3 \alpha_{n}-\pi$. But from theorem 4 , we also have that $A_{\Delta}=A_{n}=4 \pi /(2 n-4)$, hence

$$
\alpha_{n}=\frac{\pi}{3}\left(\frac{1}{1-2 / n}\right) .
$$

Finally, the expression for $\cos (a)$ becomes $\cos \left(d_{n}\right)=\left(\cos \left(\alpha_{n}\right)+\cos ^{2}\left(\alpha_{n}\right)\right) /\left(\sin ^{2}\left(\alpha_{n}\right)\right)$ in the regular case, from which the theorem follows.

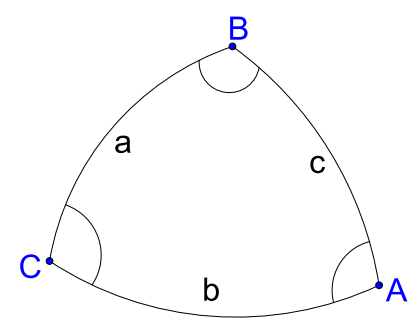

Figure 1. Illustration of a spherical triangle.

We make the following conjecture:

Conjecture 6. As $n \rightarrow \infty$, the average distance between $n$ quasi-equidistant points over the sphere is asymptotic to $d_{n}$.

Remark 7. We should also note that for fixed perimeter, the equilateral triangle is the triangle with highest area. Therefore, assuming the quasi-equilateral triangles to be actually equilateral as in the previous argument, leads to the fact that (7) should be asymptotic from below to the mean distance.

\section{Numerical method to determine quasi-equidistant points over the sphere}

In this section, we present the proposed method to obtain uniformly distributed quasi-equidistant points over the sphere. The proposed method is iterative, so we will consider that at step $p$ the distribution of points over the sphere is given by the points

$$
\hat{x}_{1}^{(p)}, \hat{x}_{2}^{(p)}, \ldots, \hat{x}_{n}^{(p)} .
$$

Our method is motivated by electric potential theory, so we consider that at each point there exists an electrical charge. By Coulomb's law, we know that the intensity $|F|$ of the electric force $F$ that a static particle at $A$ with charge $q_{A}$ induces in a static particle at $B$ with charge $q_{B}$ is given by

$$
|F|=k_{e} \frac{\left|q_{A} q_{B}\right|}{r_{A B}^{2}}
$$

where $k_{e}$ is Coulomb's constant and $r_{A B}=\|A-B\|$ is the distance between the particles $A$ and $B$. In this sense, considering without loss of generality $k_{e}\left|q_{A} q_{B}\right|=1$, we define the force vector acting on $\hat{x}_{j}^{(p)}$ by all the other (static) point charges as

$$
F_{j}^{(p)}=\sum_{k \neq j} \frac{\hat{x}_{j}^{(p)}-\hat{x}_{k}^{(p)}}{\left\|\hat{x}_{j}^{(p)}-\hat{x}_{k}^{(p)}\right\|^{2}}, \quad j=1,2, \ldots, n,
$$


and compute its tangential component at point $\hat{x}_{j}^{(p)}$ to the sphere by

$$
F_{j, t}^{(p)}=F_{j}^{(p)}-\left(F_{j}^{(p)} \cdot \hat{x}_{j}^{(p)}\right) \hat{x}_{j}^{(p)}, \quad j=1,2, \ldots, n .
$$

The vector $F_{j, t}^{(p)}$ defines the direction in which $\hat{x}_{j}^{(p)}$ should move to avoid being close to other points, so we now update the position of the points by

$$
\hat{x}_{j}^{(p+1)}=\frac{\hat{x}_{j}^{(p)}+C_{n}^{(p)} F_{j, t}^{(p)}}{\left\|\hat{x}_{j}^{(p)}+C_{n}^{(p)} F_{j, t}^{(p)}\right\|}, \quad j=1,2, \ldots, n,
$$

where $C_{n}^{(p)}$ is a dislocation constant defined at each step $p$ and dependent on the number $n$ of points. As we are working on the sphere, all distances are computed over the sphere surface.

The practical implementation of the method now depends on three factors: the initial guess, the choice of $C^{(p)}$ at each step $p$ and the stopping criteria. For the initial guess, we chose randomly distributed points of the parameterization space $[0,2 \pi] \times[0, \pi]$ that were than mapped to the sphere by a parametrization of the sphere. Though this initial guess concentrates points at the poles, it is good enough for the initial guess as one will see in section 5.1 and since it is random, does not constrain the final outcome. As for the other two factors, we will address them in more detail in the next two sections.

\subsection{Dislocation Constant}

The choice of the value of the dislocation constant is critical to achieve good results, since it should be small enough so that the system can converge to equilibrium states but large enough so that the required precision should not require too many iterations. In this sense, we considered in this paper

$$
C_{n}^{(p)}= \begin{cases}\frac{d_{n}}{20 \max _{j=1,2, \ldots, n}\left\|F_{j, t}^{(p)}\right\|}, & \text { if } \max _{j=1,2, \ldots, n}\left\|F_{j, t}^{(p)}\right\| \geq \frac{1}{d_{n}^{2}} \\ d_{n}^{3} / 8, & \text { otherwise }\end{cases}
$$

where $d_{n}$ is the estimated distance (7) over the sphere. Therefore, we are less conservative in the first steps but as the number of iterations increases and the distribution tends to a equilibrium state, we tend to consider $C_{n}^{(p)}=d_{n}^{3} / 8$, wich corresponds to the cube of the half estimated distance between points. By trial and error, this seems to be a good choice, since it guarantees a good equilibrium state.

\subsection{Stopping criteria}

There are several possibilities for the stopping criteria, as for instance, a predefined number of iterations $P$. However, this should depends on the number of points $n$, since more points should, in principle, require more iterations to achieve the equilibrium state. In this way, we considered the maximum norm of the tangential forces to be less than a given tolerance $\epsilon_{t o l}^{(n)}$ dependent on the number of points $n$, that is, $P$ is given by the smallest number of iterations such that

$$
\max _{1 \leq j \leq n}\left\|F_{j, t}^{(P)}\right\| \leq \epsilon_{t o l}^{(n)} .
$$

A small tolerance $\epsilon_{t o l}^{(n)}$ implies that the system of points is close to the equilibrium state.

Since we expect $A_{n}$ to be asymptotic to $O(1 / n)$ and therefore the distance between the points to be asymptotic to $O\left(1 / \sqrt{n}\right.$ ) (due to the relation between the side and area of an equilateral triangle of area $A_{n}$ ), having in mind that the force between neighbor charges is inversely proportional to the square of this distance, in order to have similar states of equilibrium for different values $n$, one should consider $\epsilon_{t o l}^{(n)}$ proportional to $n$. Therefore we considered $\epsilon_{t o l}^{(n)}=\frac{n}{5} \times 10^{-4}$ for the stopping criteria (9). 


\section{Direct Scattering Problem}

To illustrate the use of quasi-equidistant points for the method of fundamental solutions, we will consider the exterior time-harmonic acoustic scattering problem modelled by the Helmholtz equation

$$
\Delta u+\kappa^{2} u=0 \text { in } \mathbb{R}^{3} \backslash D
$$

where $\kappa$ is the wave number, $D$ is a bounded open domain and the total field $u$ is the sum of the known incident field $u^{i}$ and the unknown scattered field $u^{s}$, that is $u=u^{i}+u^{s}$. We will also consider the Sommerfeld radiation condition at infinity for the scattered field

$$
\lim _{r \rightarrow \infty} r\left(\frac{\partial u^{s}}{\partial r}-i \kappa u^{s}\right)=0
$$

which means that there are no energy sources at infinity, or in other words, that the scattered field is outgoing. Finally we will consider sound-soft obstacles, which means that the total field vanishes at the boundary of the obstacle $D$, that is, one has the boundary condition

$$
u=0 \text { on } \partial D .
$$

For more details, please check [21, 22]. For instance, it can be shown that the problem has a unique solution if $u^{i}$ is analytic up to the boundary of the obstacle and the obstacle is of class $C^{2}$. Again we stress that by the properties of the fundamental solution [21], an approximation of the form

$$
u^{s}(x) \approx \tilde{u}^{s}(x):=\sum_{j=1}^{n_{s}} \omega_{j} \Phi\left(x, s_{j}\right)
$$

with the source points $s_{j} \in D, j=1,2, \ldots, n_{s}$ automatically satisfies (10) and (11), regardless of the value of the weights $\omega_{j}$. Therefore, the solution of the problem might by approximated by the MFS considering collocation points $x_{k} \in \partial D, k=1,2, \ldots, n_{c}$ for the boundary condition $u^{s}\left(x_{k}\right)=-u^{i}\left(x_{k}\right)$ and solving

$$
\sum_{j=1}^{n_{s}} \omega_{j} \Phi\left(x_{k}, s_{j}\right)=-u^{i}\left(x_{k}\right), k=1,2, \ldots, n_{c},
$$

with respect to the weights $\omega_{j}, j=1,2, \ldots, n_{s}$ in a least square sense for $n_{c} \geq n_{s}$. Then the solution can be approximated in any point $x \in \mathbb{R}^{3} \backslash \bar{D}$ by (13). In section 5.2 we will illustrate numerical results on the solution by this method, considering as source and collocation points the proposed quasi-equidistant points over the sphere, with $n_{c} \geq n_{s}$.

\section{Numerical results}

In this section we will show the numerical results of the proposed method. These are two-folded: in section 5.1 we will illustrate that the method actually generates a given number of $n$ quasi-equidistant points with average distance asymptotic (from below) to the estimate (7); and in section 5.2 we illustrate that the use of these points in the context of the MFS leads to lower condition number of the linear system to be solved, in comparison with other distributions of points commonly used.

\subsection{Quasi-equidistant points}

We applied the algorithm described in section 3 to obtain $n$ uniformly distributed quasi-equidistant points over the unit sphere for $n=2^{\ell} \times 50$, with $\ell=0,1, \ldots, 6$. The set of points for each $n$ is available for download here [23]. A link to download the code will also be available on the same source reference.

In figure 2 we plot a Delaunay triangulation for the obtained points, with shorter distances in lighter color and larger distances in darker color (left) and the smaller ares in darker color and larger ares in lighter color (right), though the range of distances and areas is very small for each $n$. This Delaunay triangulation is also the basis for the computation of the average distance $\bar{d}_{\Delta}$, which is the mean of the distances of all the connections of the Delaunay triangulation. 

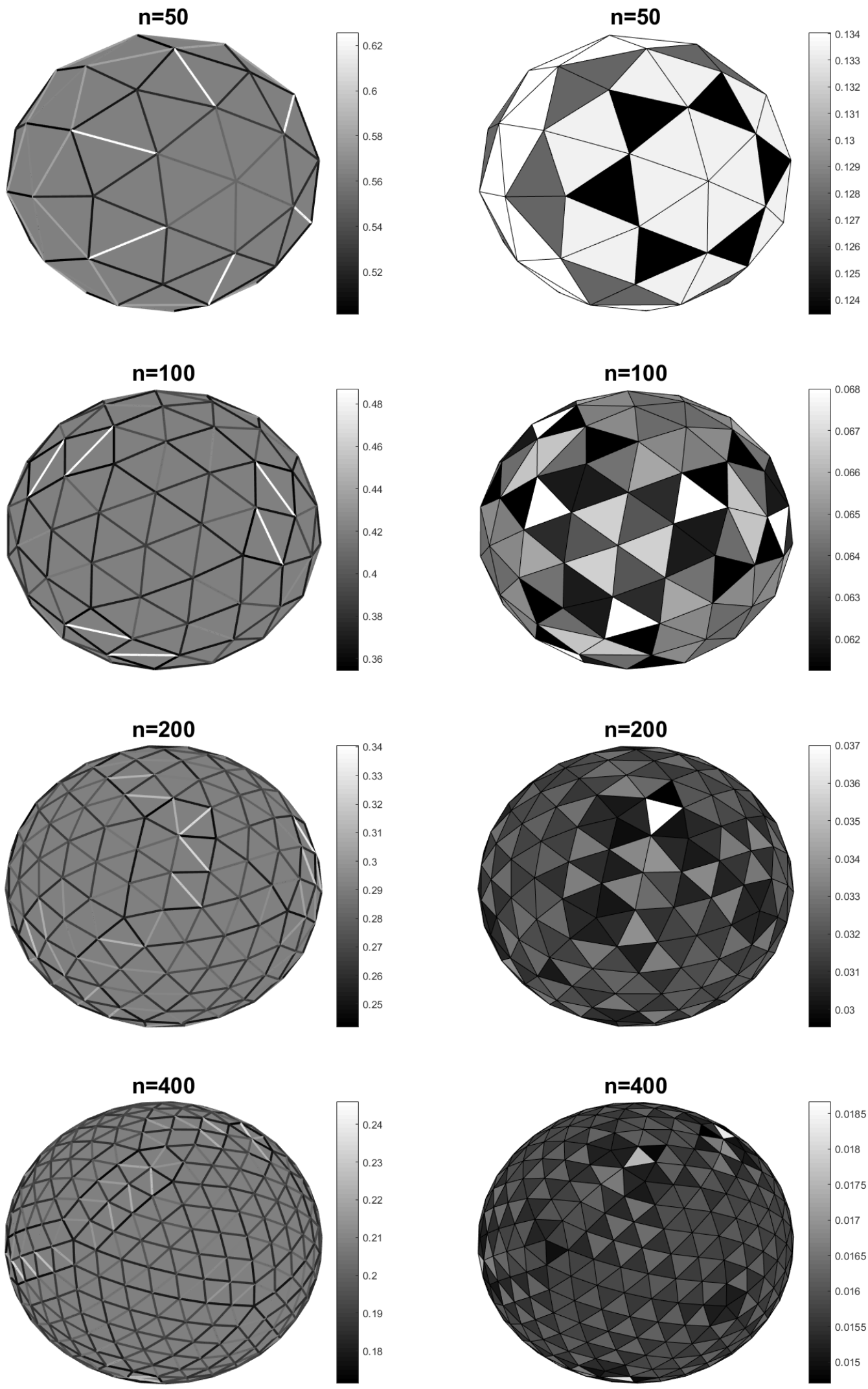

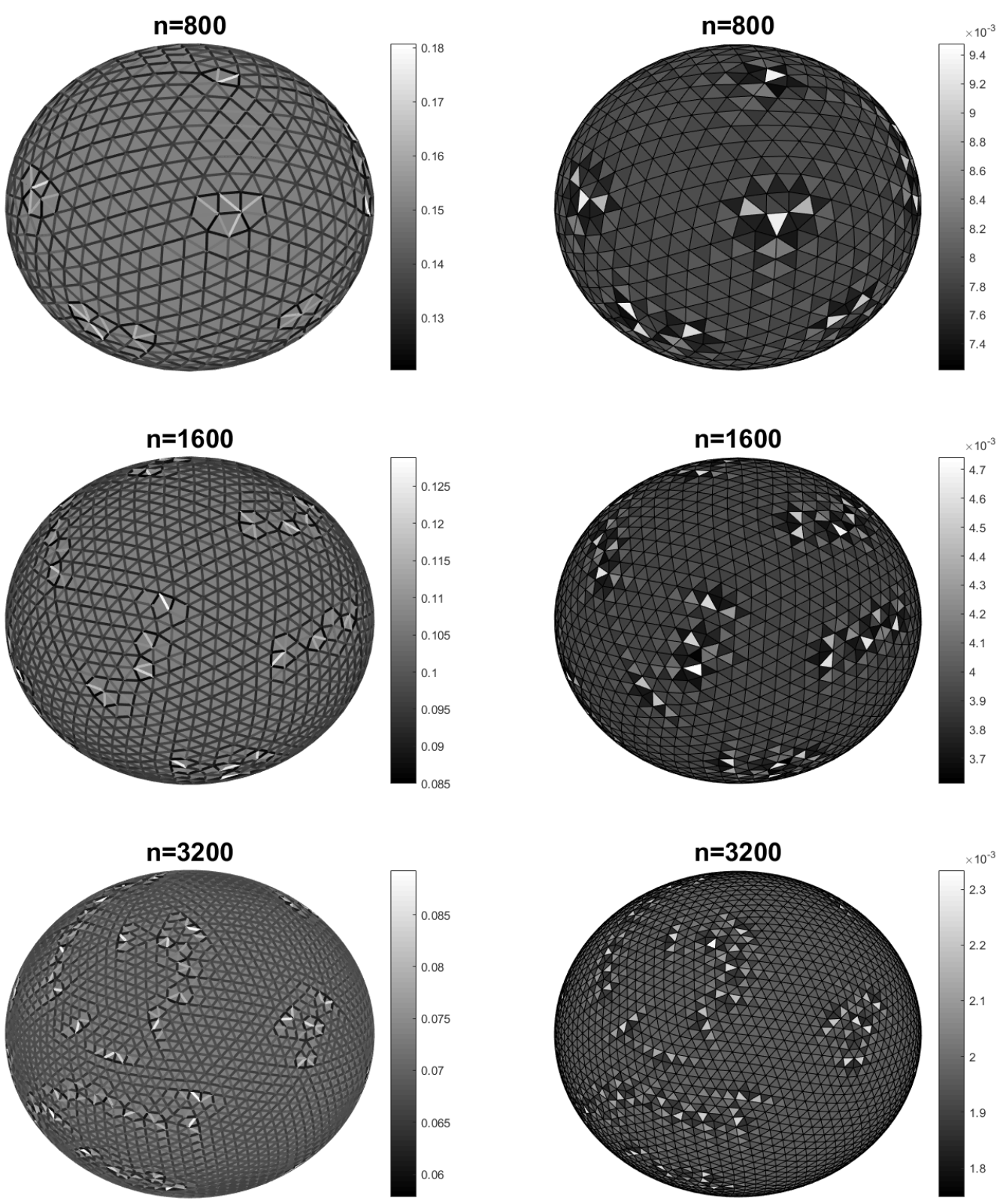

Figure 2. Plot of the color coded distances (left) between $n$ uniformly distributed quasi-equidistant points and the color coded areas (right) of the respetive Delaunay triangulation. Lighter color means higher value, however the colorbar shows that the range distances and areas is very small or each $n$. 
Table 1. Numerical results for the estimated distance $d_{n}$ by (7), the mean distance $\bar{d}_{\Delta}$ in the Delaunay triangulation for $n$ quasi-equistant points, the ratio between both, the standard deviation $\sigma_{d}(\Delta)$ of the distances given by (6) and respective variation coefficient.

\begin{tabular}{|c||c|c|c|c|c|c|c|}
\cline { 2 - 8 } \multicolumn{1}{c||}{} & $n=50$ & $n=100$ & $n=200$ & $n=400$ & $n=800$ & $n=1600$ & $n=3200$ \\
\hline$d_{n}$ & 0.5397 & 0.3813 & 0.2695 & 0.1905 & 0.1347 & 0.0952 & 0.0673 \\
\hline $\bar{d}_{\Delta}$ & 0.5430 & 0.3843 & 0.2711 & 0.1914 & 0.1352 & 0.0955 & 0.0675 \\
\hline$d_{n} / \bar{d}_{\Delta}$ & 0.9939 & 0.9922 & 0.9940 & 0.9952 & 0.9961 & 0.9969 & 0.9971 \\
\hline \hline$\sigma_{d}(\Delta)$ & 0.0363 & 0.0281 & 0.0175 & 0.0110 & 0.0073 & 0.0046 & 0.0031 \\
\hline$\sigma_{d}(\Delta) / \bar{d}_{\Delta}$ & 0.0669 & 0.0730 & 0.0644 & 0.0576 & 0.0537 & 0.0483 & 0.0459 \\
\hline
\end{tabular}

In Table 1 we show the estimated distance $d_{n}$ from (7) for $n$ points and the mean distance $\bar{h}_{n}$ between the generated $n$ uniformly distributed quasi-equistant points. We note that the ratio $d_{n} / \bar{d}_{\Delta}$ between them seems to tend to one from below, which is explained by remark 7 .

Though there is no theoretical evidence that the numerical method proposed in section 3 actually mimicks a distribution of points that leads to the theoretical estimate (7) (in the sense that it may not lead to a cover of the sphere of quasi-regular triangles), it seems that the points are uniformly distributed quasi-equidistant points and the mean distance tends to the estimate (7). Moreover the dispersion of the distances around the estimated value seems to decrease as $n$ increases, both in absolute and relative values.

Table 2. Numerical results for the estimated area $A_{n}$ by (4) (which is equal to the mean area of the triangles in the Delaunay triangulation), the standard deviation $\sigma_{A}(\Delta)$ of the distances given by (5) and respective variation coeficient.

\begin{tabular}{|c||c|c|c|c|c|c|c|}
\cline { 2 - 8 } \multicolumn{1}{c|}{} & $n=50$ & $n=100$ & $n=200$ & $n=400$ & $n=800$ & $n=1600$ & $n=3200$ \\
\hline$A_{n}$ & 0.1309 & 0.064114 & 0.031733 & 0.015787 & 0.0078737 & 0.0039319 & 0.0019647 \\
\hline \hline$\sigma_{A}(\Delta)$ & 0.0038192 & 0.001928 & 0.0010158 & 0.00043499 & 0.00024873 & 0.0001184 & $5.3524 \times 10^{-5}$ \\
\hline$\sigma_{A}(\Delta) / A_{n}$ & 0.029177 & 0.030072 & 0.03201 & 0.027554 & 0.03159 & 0.030114 & 0.027242 \\
\hline
\end{tabular}

In table 2 we present the results for the area of the triangles in the Delaunay triangulation. It is clear to see that the mean area and dispersion of the area decrease as the number of points $n$ increases. However, the relative dispersion seems to be constant, though with very low values around $3 \%$.

The distributions are also illustrated in figures 3 and 4 .

\subsection{Direct MFS solver with quasi-equidistant points}

We now compare the conditioning of the system using quasi-equidsitant points (QEP) with other choices of points, considering an obstacle $D$ as being the unit ball and a cushion shaped obstacle. For the comparison, we consider the parametrization of the sphere surface given by

$$
x(\theta, \phi)=(\cos (\theta) \sin (\phi), \sin (\theta) \sin (\phi), \cos (\phi)), \quad \theta \in[0,2 \pi], \phi \in[0, \pi] .
$$

We considered the equidistant angle (EAP) points

$$
\tilde{x}_{i j}=x\left(\tilde{\theta}_{i}, \tilde{\phi}_{j}\right), \quad i=1,2, \ldots, p, \quad j=1,2, \ldots, q,
$$

with equidistant angles $\theta_{i}=\frac{2 i \pi}{p}$ and $\tilde{\phi}_{j}=\frac{(j-1 / 2) \pi}{q}$. This distribution tends to concentrate points over the poles, as its number $n=p q$ increases. We also considered the distribution of points for the Gauss-Legendre (GLP) quadrature rule [21] given by

$$
\tilde{x}_{i j}^{(G L)}=x\left(\tilde{\theta}_{i}, \tilde{\phi}_{j}^{(G L)}\right), \quad i=1,2, \ldots, p, \quad j=1,2, \ldots, q,
$$

with the same equidistant angles $\tilde{\theta}_{i}$ and $\tilde{\phi}_{j}^{(G L)}=\arccos \left(t_{j}\right)$ with $t_{j}$ the $q$ roots of the Legendre polynomial of order $q$. It can be shown that the quadrature rule using these points is exact for spherical harmonics [24] of a certain degree. This distribution of points does not concentrate so many points over the poles, but is also far from making their distance uniform over the sphere. Both the previous approaches give rise to $n=p q$ points over the unit sphere. 

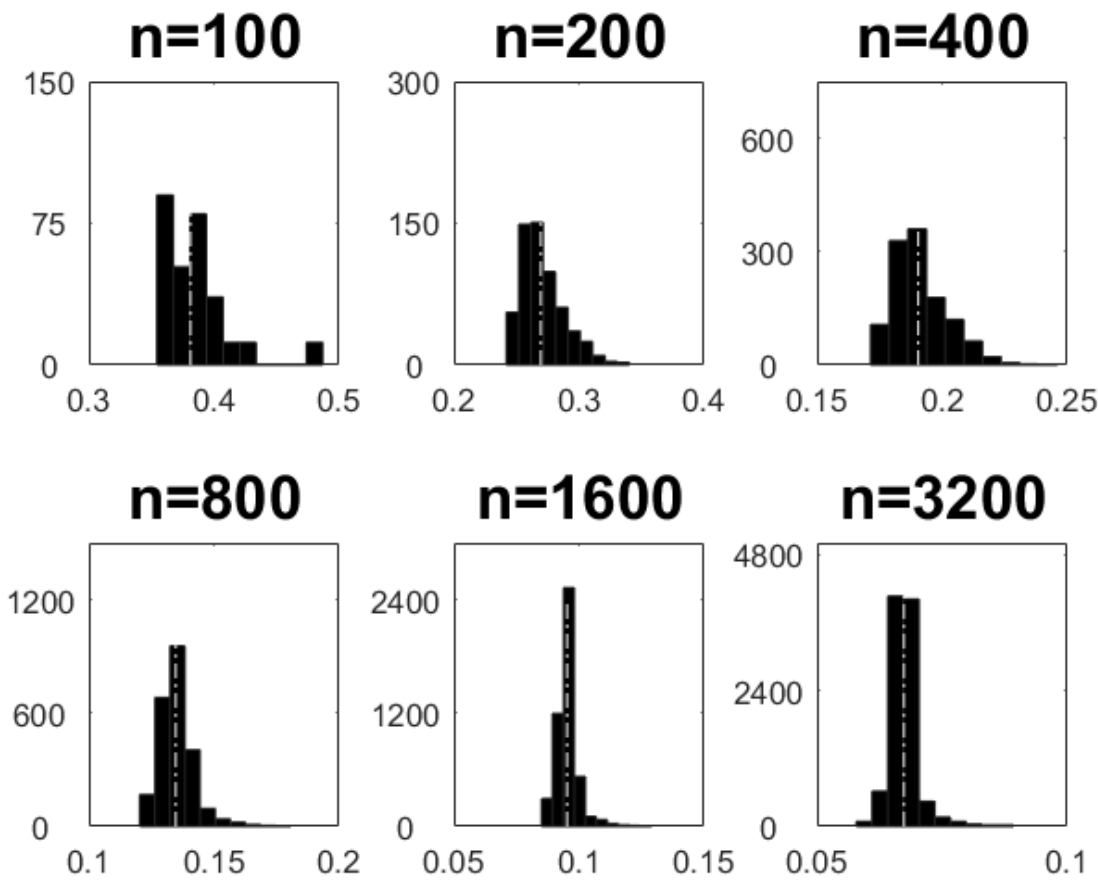

Figure 3. Histograms of the distributions of distances between points around the estimate (7) (dashed line).
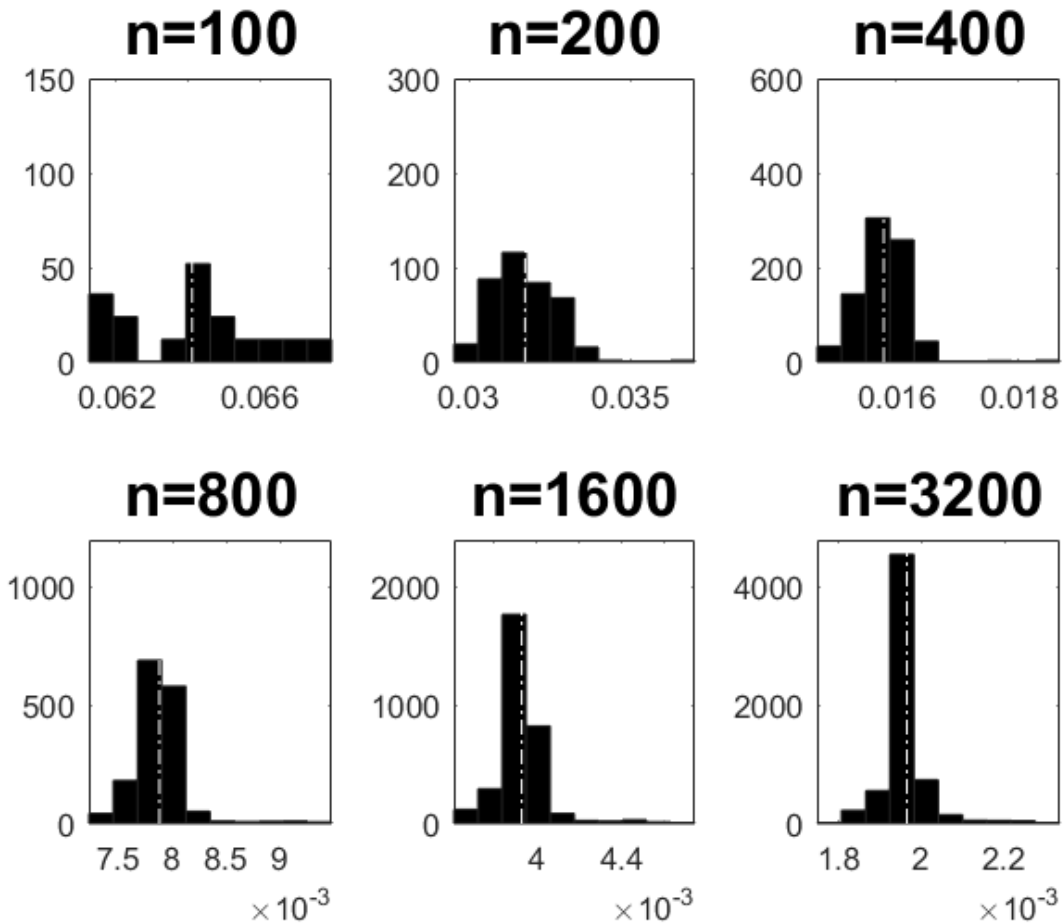

Figure 4. Histograms of the distributions of the areas for a Delaunay triangulation around the estimate (4) (dashed line). 
Following [10], we will also consider icosahedral tesselation points (ITP). These are obtained by diving each edge of the icosahedral considering $k+1(k=2,3, \ldots \ldots)$ equidistant points and then consider $(k-2)(k-1) / 2$ points in each face of the icosahedron, given by the triangular mesh defined by the previous $(k+1)$ points over the edges. The radial projection of each of these points onto the sphere gives rise to $n=10 k^{2}+2$ points over the sphere.

In the numerical examples, we considered for each set of points the MFS for $n_{c}$ points over the surface of $D$ and $n_{s}$ source points over an inner surface, obtained simply by multiplying each component of the points over the surface of $D$ by a factor of 0.9 . We also considered $n_{c} \approx 2 n_{s}$, in order to stay coherent with the previously computed points. We also considered $n_{c} \approx 2 n_{s}$, in order to stay coherent with the previously computed points in Table 1 and following results. However, due to the constraints of the number of points that can be used for EAP, GL and ITP (see the following remark 8 ), we tried to find the number of points closest to the ones used above.

Remark 8. QEP points can be obtained for any given $n$ from the proposed approach. However, EAP and GL points can only be obtained for $n$ points, where $n$ is of the form $n=p q$ with $p, q$ natural numbers. Also, ITP points can only be computed for $n$ of the form $n=10 k^{2}+2$ for some natural number $k$. This is another advantage of the proposed method for $Q E P$.

\subsubsection{Unit ball obstacle}

We will compare the performance of the proposed QEP with other commonly used points over the sphere, as mentioned in the previous lines. The design of the approach was to obtain uniformly distributed quasi-equidistant points over the unit sphere, so we will start by considering the unit sphere as the obstacle for the direct scattering problem. However, we will also see that these points can be used for star-shaped domains in the following section.

Having in mind remark 8, we first compare QEP with GLP and EAP, and then compare QEP with ITP.

It is clear from table 3 that the generated QEP by the proposed method clearly outperform the other set of GLP and EAP in terms of the conditioning of the system for the unit ball obstacle. In this way, it is clear that these points are more suitable for the MFS.

Table 3. Condition number for the unit ball obstacle considering $n_{s}$ source points and $n_{c}$ collocation points for the proposed quasi-equidistant points (QEP), the Gauss-Legendre quadrature rule points (GLP) and the parametrization equidistant angle points (EAP) with wave number $\kappa$.

\begin{tabular}{|c|c|c|c|c|c|c|}
\hline & $\begin{array}{l}n_{s}=50 \\
n_{c}=98\end{array}$ & $\begin{array}{c}n_{s}=98 \\
n_{c}=200\end{array}$ & $\begin{array}{l}n_{s}=200 \\
n_{c}=392\end{array}$ & $\begin{array}{l}n_{s}=392 \\
n_{c}=800\end{array}$ & $\begin{array}{c}n_{s}=800 \\
n_{c}=1568\end{array}$ & $\begin{array}{l}n_{s}=1568 \\
n_{c}=3200\end{array}$ \\
\hline & \multicolumn{6}{|c|}{$\kappa=0.5$} \\
\hline QEP & 24.228 & 43.075 & 96.918 & 240.22 & 922.68 & 4473.5 \\
\hline GLP & 46.548 & 261.25 & 4498.5 & 30863 & $5.651 \times 10^{8}$ & $2.163 \times 10^{14}$ \\
\hline \multirow[t]{2}{*}{ EAP } & 64.395 & 631.38 & 37045 & $1.429 \times 10^{7}$ & $2.258 \times 10^{11}$ & $6.422 \times 10^{16}$ \\
\hline & \multicolumn{6}{|c|}{$\kappa=1$} \\
\hline QEP & 21.460 & 38.482 & 86.893 & 215.79 & 829.50 & 4024.0 \\
\hline GLP & 41.638 & 234.23 . & 4038.6 & 27726 & $5.080 \times 10^{8}$ & $1.945 \times 10^{14}$ \\
\hline \multirow[t]{2}{*}{ EAP } & 57.755 & 567.40 & 33323 & $1.286 \times 10^{7}$ & $2.033 \times 10^{11}$ & $5.738 \times 10^{16}$ \\
\hline & \multicolumn{6}{|c|}{$\kappa=2$} \\
\hline QEP & 12.488 & 23.171 & 53.083 & 132.84 & 512.34 & 2491.1 \\
\hline GLP & 30.466 & 180.67 & 3231.1 & 22697 & $4.230 \times 10^{8}$ & $1.639 \times 10^{14}$ \\
\hline \multirow[t]{2}{*}{ EAP } & 48.541 & 483.96 & 28646 & $1.107 \times 10^{7}$ & $1.755 \times 10^{11}$ & $4.802 \times 10^{16}$ \\
\hline & \multicolumn{6}{|c|}{$K=5$} \\
\hline QEP & 15.874 & 36.290 & 117.16 & 178.69 & 252.16 & 1245.9 \\
\hline GLP & 60.615 & 268.69 & 2098.2 & 15607 & $3.033 \times 10^{8}$ & $1.204 \times 10^{14}$ \\
\hline EAP & 50.165 & 348.63 & 21448 & $8.329 \times 10^{6}$ & $1.337 \times 10^{11}$ & $3.536 \times 10^{16}$ \\
\hline
\end{tabular}


We also compared the performance of the proposed QEP with ITP. In table 4 we compare the performance of these ITP with comparison to the proposed QEP, showing that for larger $n$ QEP perform better in terms of the conditioning of the system, though both condition numbers are of similar magnitude.

Table 4. Condition number for the unit ball obstacle considering $n_{s}$ source points and $n_{c}$ collocation points for the proposed quasi-equidistant points (QEP) and Icosahedral tesselation points (ITP) with wave number $\kappa$.

\begin{tabular}{|c|c|c|c|c|c|c|}
\hline & $\begin{array}{l}n_{s}=42 \\
n_{c}=92\end{array}$ & $\begin{array}{c}n_{s}=92 \\
n_{c}=162\end{array}$ & $\begin{array}{l}n_{s}=162 \\
n_{c}=362\end{array}$ & $\begin{array}{l}n_{s}=362 \\
n_{c}=812\end{array}$ & $\begin{array}{c}n_{s}=812 \\
n_{c}=1692\end{array}$ & $\begin{array}{l}n_{s}=1692 \\
n_{c}=3242\end{array}$ \\
\hline & \multicolumn{6}{|c|}{$\kappa=0.5$} \\
\hline QEP & 20.578 & 42.45 & 72.58 & 211.32 & 912.65 & 5541.5 \\
\hline \multirow[t]{2}{*}{ ITP } & 18.038 & 39.723 & 68.166 & 218.76 & 1125.6 & 8938.4 \\
\hline & \multicolumn{6}{|c|}{$\kappa=1$} \\
\hline QEP & 18.22 & 37.9 & 65.029 & 189.8 & 820.53 & 4985.0 \\
\hline \multirow[t]{2}{*}{ ITP } & 15.905 & 35.439 & 61.08 & 196.47 & 1011.9 & 8039.7 \\
\hline & \multicolumn{6}{|c|}{$\kappa=2$} \\
\hline QEP & 10.566 & 22.765 & 39.623 & 116.77 & 506.93 & 3086.7 \\
\hline \multirow[t]{2}{*}{ ITP } & 9.0568 & 21.219 & 37.22 & 120.82 & 624.81 & 4974.7 \\
\hline & \multicolumn{6}{|c|}{$\kappa=5$} \\
\hline QEP & 11.059 & 47.007 & 75.031 & 172.73 & 249.91 & 1545.8 \\
\hline ITP & 15.592 & 65.593 & 134.2 & 207.29 & 333.66 & 2695.3 \\
\hline
\end{tabular}

The total fields $u$ are plotted in figure 5, generated by an obstacle $D$ being the unit ball and considering as incident field an incident plane wave

$$
u^{i}(x)=e^{i \kappa x \cdot v}
$$

for the incident direction $v=\left(\frac{\sqrt{2}}{2}, 0, \frac{\sqrt{2}}{2}\right)$ and several wave number $\kappa$.

As for accuracy, we have computed the numerical the maximum norm and $L^{2}$-norm of the aproximated total field $\tilde{u}=u^{i}+\tilde{u}^{s}$ over the sphere though the Gauss-Legendre quadrature rule with 3200 points, that should be zero according to que boundary condition (12). In this way, Tables 5 and 6illustrate that as the number of points increases, the accuracy of the method also increases, since the norm of the aproximated total field $\tilde{u}=u^{i}+\tilde{u}^{s}$ tends to fulfill the boundary condition (12). As the wave number $\kappa$ increases, it is expected that a higher number of points is required to obtain the same level of accuracy in the aproximation, as illustrated in Tables 5 and 6.

Table 5. Numerical $L^{2}$-norm of the aproximated total field $\tilde{u}=u^{i}+\tilde{u}^{s}$ though the Gauss-Legendre quadrature rule.

\begin{tabular}{|c||c|c|c|c|c|c|}
\multicolumn{1}{l|}{} & $n_{s}=50$ & $n_{s}=100$ & $n_{s}=200$ & $n_{s}=400$ & $n_{s}=800$ & $n_{s}=1600$ \\
& $n_{c}=100$ & $n_{c}=200$ & $n_{c}=400$ & $n_{c}=800$ & $n_{c}=1600$ & $n_{c}=3200$ \\
\hline$\kappa=0.5$ & 0.14138 & $5.4486 \times 10^{-2}$ & $1.6395 \times 10^{-2}$ & $3.6171 \times 10^{-3}$ & $5.1690 \times 10^{-4}$ & $3.8959 \times 10^{-5}$ \\
\hline$K=1$ & 0.19476 & $7.5645 \times 10^{-2}$ & $2.2647 \times 10^{-2}$ & $5.0139 \times 10^{-3}$ & $7.1661 \times 10^{-4}$ & $5.3603 \times 10^{-5}$ \\
\hline$K=2$ & 0.30807 & 0.11948 & $3.5486 \times 10^{-2}$ & $7.8387 \times 10^{-3}$ & $1.1205 \times 10^{-3}$ & $8.3476 \times 10^{-5}$ \\
\hline$K=5$ & 1.0595 & 0.65808 & 0.59088 & 0.29744 & $4.5922 \times 10^{-2}$ & $3.3826 \times 10^{-3}$ \\
\hline
\end{tabular}

\subsubsection{Cushion shaped obtacle}

These QEP points can easily be mapped to 3D star-shaped surfaces, that is, to surfaces that can be expressed as

$$
\{r(\theta, \phi) x(\theta, \phi): \theta \in[0,2 \pi], \phi \in[0, \pi]\}
$$




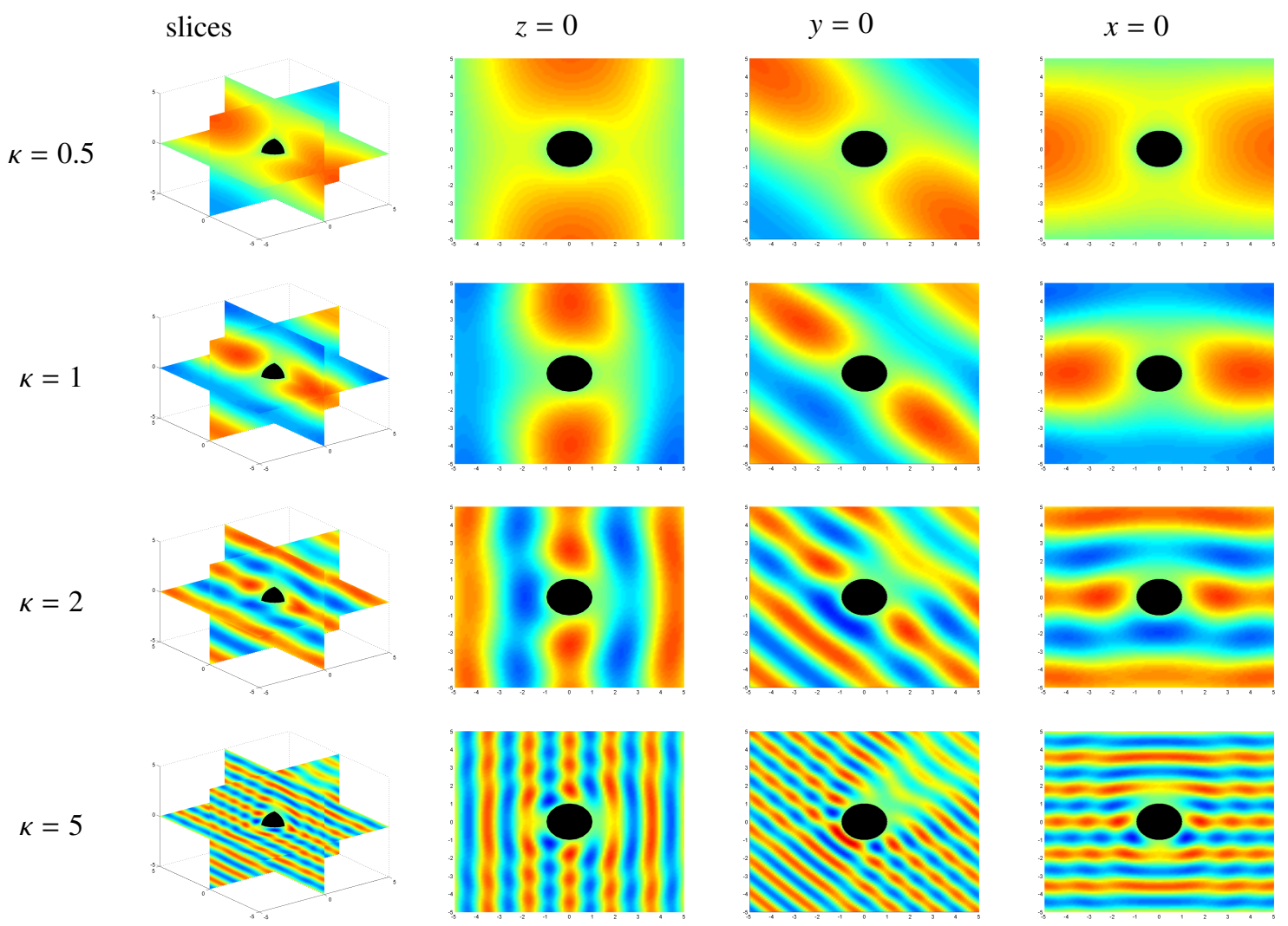

Figure 5. Approximation of the total field for the unit ball obstacle for several values of wave number $\kappa$ by the MFS, considering $n_{s}=1568$ source points and $n_{c}=3200$ collocation points.

Table 6. Maximum norm of the absolute aproximated total field $|\tilde{u}|=\left|u^{i}+\tilde{u}^{s}\right|$ in Gauss-Legendre points.

\begin{tabular}{|c||c|c|c|c|c|c|}
\multicolumn{1}{l|}{} & $n_{s}=50$ & $n_{s}=100$ & $n_{s}=200$ & $n_{s}=400$ & $n_{s}=800$ & $n_{s}=1600$ \\
& $n_{c}=100$ & $n_{c}=200$ & $n_{c}=400$ & $n_{c}=800$ & $n_{c}=1600$ & $n_{c}=3200$ \\
\hline$K=0.5$ & 0.23161 & $8.3400 \times 10^{-2}$ & $1.9552 \times 10^{-2}$ & $4.5091 \times 10^{-3}$ & $6.8786 \times 10^{-4}$ & $7.5632 \times 10^{-5}$ \\
\hline$K=1$ & 0.33830 & 0.12541 & $2.9474 \times 10^{-2}$ & $6.7272 \times 10^{-3}$ & $1.0061 \times 10^{-3}$ & $1.1524 \times 10^{-4}$ \\
\hline$K=2$ & 0.53790 & 0.20114 & $4.8666 \times 10^{-2}$ & $1.0764 \times 10^{-2}$ & $1.5731 \times 10^{-3}$ & $1.8875 \times 10^{-4}$ \\
\hline$K=5$ & 1.0871 & 0.50931 & 0.49323 & 0.38543 & $6.5385 \times 10^{-2}$ & $8.0281 \times 10^{-3}$ \\
\hline
\end{tabular}

with a real-valued non-negative continuous function $r$ and $x$ as in (14). To illustate it, we consider a cushion shaped obstacle defined by

$$
r(\theta, \phi)=\sqrt{0.8+0.5(\cos (2 \phi)-1)(\cos (4 \theta)-1)}
$$

As it is clear from tables 7 and 8 , the results are very similar for the cushion shaped obstacle. The generated QEP by the proposed method also clearly outperform the set of GLP and EAP in terms of the conditioning of the system for the cushion shaped obstacle, specially with the increase of the number $n$ of points used. As for the comparison with ITP, the conditionng of the system for the proposed QEP is also better for larger $n$, though with the same order of magnitude. Again, this illustrates that the proposed QEP points are more suitable for the MFS.

The total fields $u$ generated by the cushion shaped obstacle with the same incident direction $v$ are plotted in figure 6 for different wave number $\kappa$. 
Table 7. Condition number for the cushion shaped obstacle considering $n_{s}$ source points and $n_{c}$ collocation points for the proposed quasi-equidistant points (QEP) and the Gauss-Legendre quadrature rule points (GL) and the parametrization equidistant angle points (EA) with wave number $\kappa$.

\begin{tabular}{|c|c|c|c|c|c|c|}
\hline & $\begin{array}{l}n_{s}=50 \\
n_{c}=98\end{array}$ & $\begin{array}{c}n_{s}=98 \\
n_{c}=200\end{array}$ & $\begin{array}{l}n_{s}=200 \\
n_{c}=392\end{array}$ & $\begin{array}{l}n_{s}=392 \\
n_{c}=800\end{array}$ & $\begin{array}{c}n_{s}=800 \\
n_{c}=1568\end{array}$ & $\begin{array}{l}n_{s}=1568 \\
n_{c}=3200\end{array}$ \\
\hline & \multicolumn{6}{|c|}{$\kappa=0.5$} \\
\hline QEP & 30.905 & 46.853 & 113.69 & 216.47 & 750.70 & 3829.5 \\
\hline GLP & 26.688 & 123.40 & 1194.9 & 70849 & $8.759 \times 10^{7}$ & $7.168 \times 10^{12}$ \\
\hline \multirow[t]{2}{*}{ EAP } & 30.833 & 309.89 & 4738.8 & $2.134 \times 10^{6}$ & $1.654 \times 10^{10}$ & $1.606 \times 10^{16}$ \\
\hline & \multicolumn{6}{|c|}{$\kappa=1$} \\
\hline QEP & 26.230 & 40.168 & 99.207 & 189.50 & 658.03 & 3359.3 \\
\hline GLP & 22.631 & 110.01 & 1067.5 & 63551 & $7.873 \times 10^{7}$ & $6.451 \times 10^{12}$ \\
\hline \multirow[t]{2}{*}{ EAP } & 27.651 & 279.46 & 4267.1 & $1.927 \times 10^{6}$ & $1.494 \times 10^{10}$ & $1.495 \times 10^{16}$ \\
\hline & \multicolumn{6}{|c|}{$\kappa=2$} \\
\hline QEP & 14.069 & 22.204 & 58.984 & 114.02 & 398.16 & 2038.9 \\
\hline GLP & 16.027 & 92.714 & 921.98 & 56257 & $7.064 \times 10^{7}$ & $5.835 \times 10^{12}$ \\
\hline \multirow[t]{2}{*}{ EAP } & 25.113 & 256.47 & 3896.3 & $1.778 \times 10^{6}$ & $1.381 \times 10^{10}$ & $1.257 \times 10^{16}$ \\
\hline & \multicolumn{6}{|c|}{$\kappa=5$} \\
\hline QEP & 11.014 & 20.900 & 55.230 & 91.294 & 218.43 & 1146.1 \\
\hline GLP & 11.955 & 61.223 & 644.08 & 42910 & $5.1682 \times 10^{7}$ & $3.9071 \times 10^{12}$ \\
\hline EAP & 17.369 & 197.04 & 2927.0 & $1.4415 \times 10^{6}$ & $9.2056 \times 10^{9}$ & $7.6881 \times 10^{15}$ \\
\hline
\end{tabular}

Table 8. Condition number for the cushion shaped obstacle considering $n_{s}$ source points and $n_{c}$ collocation points for the proposed quasi-equidistant points (QEP) and Icosahedral tesselation points (ITP) with wave number $\kappa$.

\begin{tabular}{|c|c|c|c|c|c|c|}
\hline & $\begin{array}{l}n_{s}=42 \\
n_{c}=92\end{array}$ & $\begin{array}{c}n_{s}=92 \\
n_{c}=162\end{array}$ & $\begin{array}{l}n_{s}=162 \\
n_{c}=362\end{array}$ & $\begin{array}{l}n_{s}=362 \\
n_{c}=812\end{array}$ & $\begin{array}{c}n_{s}=812 \\
n_{c}=1692\end{array}$ & $\begin{array}{l}n_{s}=1692 \\
n_{c}=3242\end{array}$ \\
\hline & \multicolumn{6}{|c|}{$\kappa=0.5$} \\
\hline QEP & 27.803 & 57.877 & 104.09 & 279.91 & 1346.5 & 8158.6 \\
\hline \multirow[t]{2}{*}{ ITP } & 24.215 & 60.603 & 85.868 & 267.06 & 1299.7 & 9860.5 \\
\hline & \multicolumn{6}{|c|}{$\kappa=1$} \\
\hline QEP & 22.842 & 49.293 & 89.926 & 243.9 & 1177.3 & 7144.4 \\
\hline \multirow[t]{2}{*}{ ITP } & 20.271 & 52.220 & 74.390 & 233.07 & 1137.9 & 8645.5 \\
\hline & \multicolumn{6}{|c|}{$\kappa=2$} \\
\hline QEP & 10.994 & 26.375 & 51.328 & 144.07 & 705.01 & 4305.8 \\
\hline \multirow[t]{2}{*}{ ITP } & 10.186 & 29.408 & 42.953 & 138.78 & 686.35 & 5245.1 \\
\hline & \multicolumn{6}{|c|}{$\kappa=5$} \\
\hline QEP & 7.6092 & 12.377 & 17.088 & 66.089 & 359.06 & 2298.5 \\
\hline ITP & 8.1682 & 14.398 & 17.651 & 70.805 & 385.79 & 3075.1 \\
\hline
\end{tabular}

\section{Conclusions and Future Work}

As illustrated, the proposed method generates uniformly distributed quasi-equidistant points, whose distances are distributed around the conjectured estimate (7) with small deviations. Moreover, the area of the triangles of the corresponding Delaunay triangulation is also distributed around the estimated value (4) with very small dispersion. 


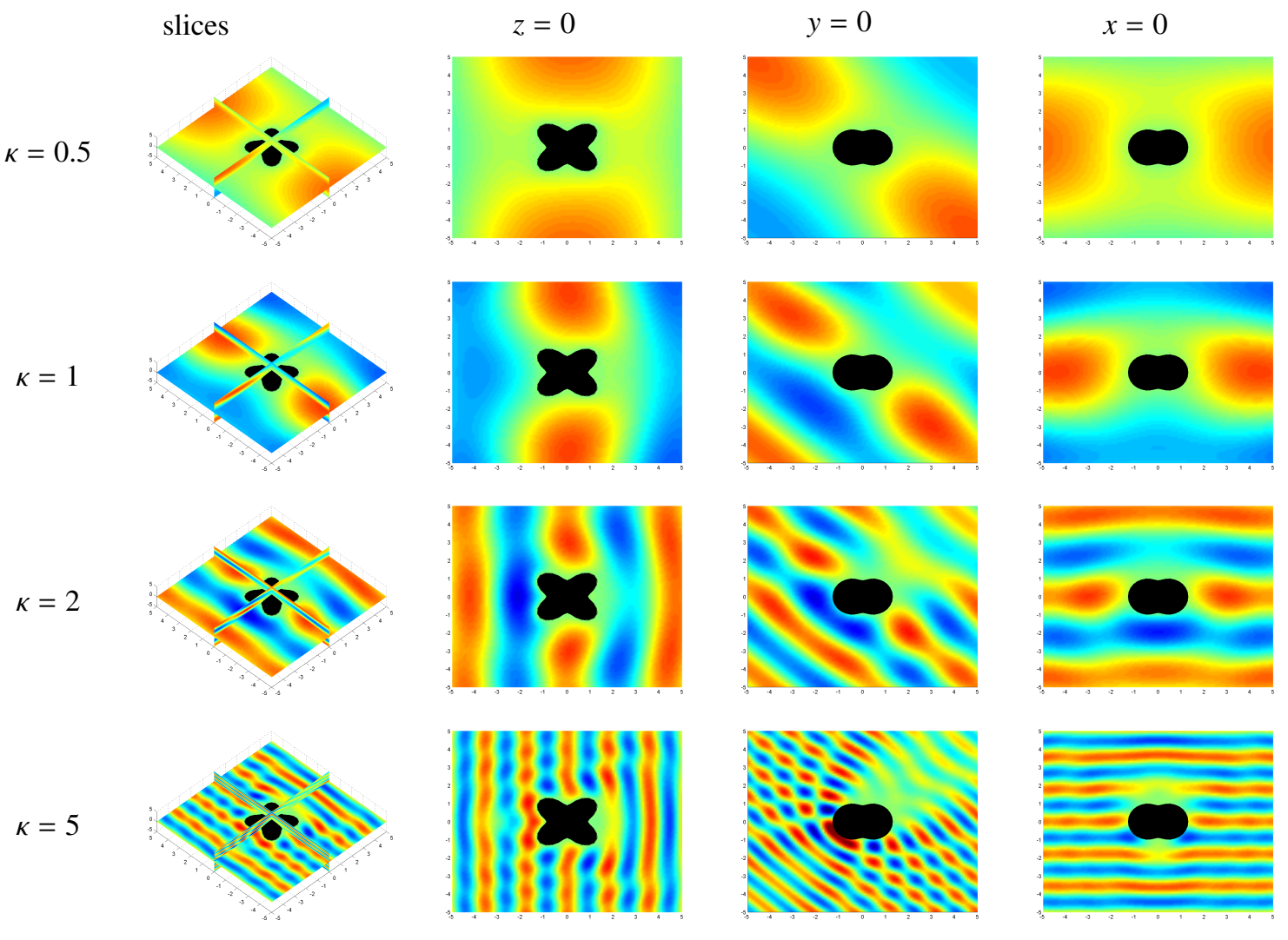

Figure 6. Approximation of the total field for the cushion shaped obstacle for several values of wave number $\kappa$ by the MFS, considering $n_{s}=1568$ source points and $n_{c}=3200$ collocation points.

In the context of scattering problems and time-harmonic wave propagation, the proposed distribution of points leads to high accuracy with a much smaller condition number (in comparison to commonly used points) for linear systems generated from the method of fundamental solutions, which means that its use is beneficial for these numerical methods. When compared to the icosahedral tesselation points (ITP), the proposed points also perform better in terms of conditioning, especially for a large number of points. Moreover, the proposed method can be applied to any natural number of points $n$, while by construction the ITP are restricted to a number of points of the form $n=10 k^{2}+2$ for some natural $k$.

As shown, the proposed points can be used for scattering problems for star-shaped domains, maintaining the good conditioning property, though the quasi-equidistant feature is lost due to the different values of the curvature over the surface. The generalization to other surfaces is a subject for future work.

\section{Aknowledgements}

The first author aknowledges his work is partially supported by UID/Multi/04019/2013. The second author aknowledges his work is partially supported by UID/Multi/04621/2013 and by FCT (Portugal) research project PTDC/EMD-EMD/32162/2017.

\section{References}

[1] C. J. S. Alves, P. R. S. Antunes, The method of fundamental solutions applied to some inverse eigenproblems, SIAM Journal on Scientific Computing 35 (3) (2013) A1689-A1708. arXiv: https://doi.org/10.1137/110860380, doi:10.1137/110860380. URL https://doi.org/10.1137/110860380 
[2] G. Ala, G. Fasshauer, E. Francomano, S. Ganci, M. McCourt, The method of fundamental solutions in solving coupled boundary value problems for m/eeg, SIAM Journal on Scientific Computing 37 (4) (2015) B570-B590. arXiv:https://doi.org/10.1137/ 13094921X, doi:10.1137/13094921X.

URL https: / / doi.org/10.1137/13094921x

[3] A. Kleefeld, L. Pieronek, The method of fundamental solutions for computing acoustic interior transmission eigenvalues, Inverse Problems 34 (3) (2018) 035007

URL http://stacks.iop.org/0266-5611/34/i=3/a=035007

[4] L. Marin, A. Karageorghis, D. Lesnic, Regularized \{MFS $\}$ solution of inverse boundary value problems in three-dimensional steady-state linear thermoelasticity, International Journal of Solids and Structures 91 (2016) 127 - 142. doi: http://dx.doi.org/10.1016/j. ijsolstr.2016.03.013.

URL http://www.sciencedirect.com/science/article/pii/s0020768316001323

[5] Y.-S. Smyrlis, Applicability and applications of the method of fundamental solutions, Mathematics of Computation 78 (267) (2009) 13991434.

URL http: / /www.jstor.org/stable/40234665

[6] C. J. Alves, On the choice of source points in the method of fundamental solutions, Engineering Analysis with Boundary Elements 33 (12) (2009) 1348 - 1361, special Issue on the Method of Fundamental Solutions in honour of Professor Michael Golberg. doi : http://dx . doi.org/10.1016/j.enganabound.2009.05.007.

URL http://www.sciencedirect.com/science/article/pii/s0955799709001301

[7] A. Barnett, T. Betcke, Stability and convergence of the method of fundamental solutions for helmholtz problems on analytic domains, Journal of Computational Physics 227 (14) (2008) 7003-7026. doi: http://dx. doi.org/10.1016/j.jcp.2008.04.008. URL http://www.sciencedirect.com/science/article/pii/s0021999108002106

[8] Y.-S. Smyrlis, A. Karageorghis, Efficient implementation of the mfs: The three scenarios, Journal of Computational and Applied Mathematics 227 (1) (2009) 83-92, special Issue of Proceedings of \{NUMAN\} 2007 Conference: Recent Approaches to Numerical Analysis: Theory, Methods and Applications. doi:http://dx.doi.org/10.1016/j.cam.2008.07.010.

URL http: //www.sciencedirect.com/science/article/pii/s0377042708003312

[9] D. Young, C. Tsai, C. Chen, C. Fan, The method of fundamental solutions and condition number analysis for inverse problems of laplace equation, Computers \& Mathematics with Applications 55 (6) (2008) 1189-1200. doi:https://doi.org/10.1016/j.camwa. 2007.05 .015 . URL http: //www.sciencedirect.com/science/article/pii/s0898122107005883

[10] C. J. Alves, S. S. Valtchev, Numerical comparison of two meshfree methods for acoustic wave scattering, Engineering Analysis with Boundary Elements 29 (4) (2005) 371 - 382, mesh Reduction Methods - Part \{IIIMesh $\}$ Reduction Methods - Part $\{$ III $\}$. doi : http: //dx. doi. org/10.1016/j.enganabound.2004.09.008.

URL http://www.sciencedirect.com/science/article/pii/s0955799705000196

[11] P. R. S. Antunes, A numerical algorithm to reduce ill-conditioning in meshless methods for the helmholtz equation, Numerical Algorithmsdoi:10.1007/s11075-017-0465-z. URL https: //doi.org/10.1007/s11075-017-0465-z

[12] C. S. Chen, A. Karageorghis, Y. Li, On choosing the location of the sources in the mfs, Numerical Algorithms 72 (1) (2016) 107-130. doi: $10.1007 /$ s11075-015-0036-0. URL https://doi.org/10.1007/s11075-015-0036-0

[13] C. J. Alves, P. R. Antunes, The method of fundamental solutions applied to boundary value problems on the surface of a sphere, Computers \& Mathematics with Applications 75 (7) (2018) 2365 -2373. doi : https: / doi.org/10.1016/j.camwa.2017.12.015. URL http://www.sciencedirect.com/science/article/pii/s0898122117307782

[14] P. R. S. Antunes, Numerical calculation of eigensolutions of 3d shapes using the method of fundamental solutions, Numerical Methods for Partial Differential Equations 27 (6) (2011) 1525-1550. doi:10.1002/num.20594. URL http://dx.doi.org/10.1002/num.20594

[15] B. Bin-Mohsin, D. Lesnic, The method of fundamental solutions for helmholtz-type equations in composite materials, Computers \& Mathematics with Applications 62 (12) (2011) 4377 -4390. doi : http://dx.doi.org/10.1016/j.camwa.2011.10.006. URL http: //www.sciencedirect.com/science/article/pii/s0898122111008704

[16] T. J. Chen, H. M. Chang, H. K. Chen, L. I. Chen, Boundary collocation method for acoustic eigenanalysis of three-dimensional cavities using radial basis function, Computational Mechanics 29 (4) (2002) 392-408. doi:10.1007/s00466-002-0350-y. URL http://dx.doi.org/10.1007/s00466-002-0350-y

[17] G. S. Fam, Y. F. Rashed, The method of fundamental solutions applied to $3 \mathrm{~d}$ elasticity problems using a continuous collocation scheme, Engineering Analysis with Boundary Elements 33 (3) (2009) 330-341. doi : http://dx.doi .org/10.1016/j.enganabound. 2008.07 .002 .

URL http: //www.sciencedirect.com/science/article/pii/s0955799708001215

[18] A. Karageorghis, D. Lesnic, L. Marin, The method of fundamental solutions for three-dimensional inverse geometric elasticity problems, Computers \& Structures 166 (2016) 51 -59. doi : http://dx.doi.org/10.1016/j.compstruc.2016.01.010. URL http://www.sciencedirect.com/science/article/pii/s0045794916000110

[19] G. Ala, E. Francomano, G. E. Fasshauer, S. Ganci, M. J. McCourt, A meshfree solver for the meg forward problem, IEEE Transactions on Magnetics 51 (3) (2015) 1-4. doi:10.1109/TMAG.2014.2356134.

[20] G. Ala, G. E. Fasshauer, E. Francomano, S. Ganci, M. J. McCourt, An augmented mfs approach for brain activity reconstruction, Mathematics and Computers in Simulation 141 (2017) 3 - 15, new Trends in Numerical Analysis: Theory, Methods, Algorithms and Applications NETNA 2015 (dedicated to Professor F.A. Costabile on his 70th birthday) held in Falerna (CZ), Italy during June 18-20, 2015. doi: https://doi.org/10.1016/j.matcom.2016.11.009. URL http: //www.sciencedirect.com/science/article/pii/s0378475416302488 
[21] D. Colton, R. Kress, Inverse Acoustic and Electromagnetic Scattering Theory, 3rd Edition, Springer, 2013.

[22] P. Serranho, A hybrid method for inverse scattering for sound-soft obstacles in 3D, Inverse Problems and Imaging 4 (2007) 691-712.

[23] A. Aráujo, P. Serranho, Quasi-equidistant points for download:, http://www . univ-ab.pt/ pserranho/Pts.Sphere.

[24] M. Ganesh, I. Graham, A high order algorithm for obstacle scattering in three dimensions, J. Comp. Phys. 198 (2004) 211-242. 